\title{
Veias linfonodais: uma causa pouco conhecida de varizes
}

\author{
Lymph node veins: a little-known cause of varicose veins
}

\author{
André Paciello Romualdo, Roberto de Moraes Bastos, Alessandro Cappucci, Mathias Fatio, \\ Andréa Tsunoda, Pollyanna Campos, Alberto Lobo Machado, Eduardo Hideki Tokura*
}

\begin{abstract}
Resumo
As veias linfonodais fazem parte de uma rede venosa no triângulo de Scarpa, que liga em vários pontos o sistema venoso superficial às veias profundas, e podem tanto ser causa de incompetência do sistema venoso superficial quanto estar envolvidas na recorrência pós-safenectomia. Na rotina diária dos exames de Doppler venoso de membros inferiores, temos notado de maneira cada vez mais freqüente a associação das veias linfonodais com varizes primárias e recorrentes. A adequada caracterização dessas veias pode ajudar na compreensão do mecanismo fisiopatológico do aparecimento das varizes e permitir um controle e tratamento mais dirigidos. Este artigo lança luz sobre os aspectos anatômicos e fisiológicos das veias linfonodais, objetivando chamar a atenção dos profissionais envolvidos no diagnóstico de doenças venosas dos membros inferiores para uma causa pouco difundida de varizes.
\end{abstract}

Palavras-chave: Safena, Doppler, varizes.

\section{Introdução}

O exame ultra-sonográfico com Doppler colorido tem um papel fundamental no diagnóstico e no seguimento das variadas doenças vasculares, particularmente no estudo venoso dos membros inferiores.

Tratamos neste artigo de causa pouco conhecida de varizes, as veias linfonodais, que tanto podem estar relacionadas à forma primária quanto aos casos de recorrência da doença varicosa. Tais veias têm sido identificadas com freqüência cada vez maior pelos médicos que trabalham com diagnósticos vasculares, daí a importância do seu conhecimento como possível fator etiológico da doença descrita.

\section{Veias linfonodais: aspectos anatômicos}

A junção safeno-femoral pode ser definida como o segmento venoso femoral delimitado pelas valvas supra

\begin{abstract}
Lymph node veins are part of a venous network in Scarpa's triangle, communicating in many points the superficial venous system and the deep veins, and may either be the cause of incompetence of the superficial venous system, or be involved in recurrent varicose veins after saphenous vein stripping. In the daily routine of venous Doppler examination of the lower extremities, an increasingly frequent association of lymph node veins with primary and/or recurrent varicose veins has been noticed. Appropriate characterization of these veins may help to understand the pathophysiological mechanism of varicose vein appearance and provide a more focused approach to follow-up and treatment. This article sheds some light on the anatomical and physiological aspects of lymph node veins, drawing the attention of professionals involved in the diagnosis of venous disorders of the lower extremities to a little-known cause of varicose veins.
\end{abstract}

Keywords: Saphenous, Doppler, varicose veins.

e infra-safena, bem como pelas valvas terminal e préterminal da crossa da veia safena magna ${ }^{1,2}$. As tributárias proximais que drenam entre as valvas pré-terminal e terminal são as veias ilíaca circunflexa superficial (trajeto lateral), epigástrica superficial (trajeto cranial) e pudenda externa (trajeto medial). Essas veias podem ser únicas ou múltiplas, podem drenar isoladamente ou em tronco único, e ainda terminar diretamente na veia femoral comum. Tais veias têm importância clínica porque podem ser fonte de refluxo da safena magna nos casos de valvas terminais competentes e pré-terminais incompetentes (Figuras 1 e 2). As tributárias distais são as veias safena acessória anterior (trajeto lateral), que encontra a safena magna entre as valvas terminal e pré-terminal, e a safena acessória posterior (trajeto medial), drenando freqüentemente distalmente à valva pré-terminal $^{1-3}$.

* Fleury - Medicina e Saúde, São Paulo, SP.

Não foram declarados conflitos de interesse associados à publicação deste artigo.

Artigo submetido em 24.06.08, aceito em 14.10.08. 


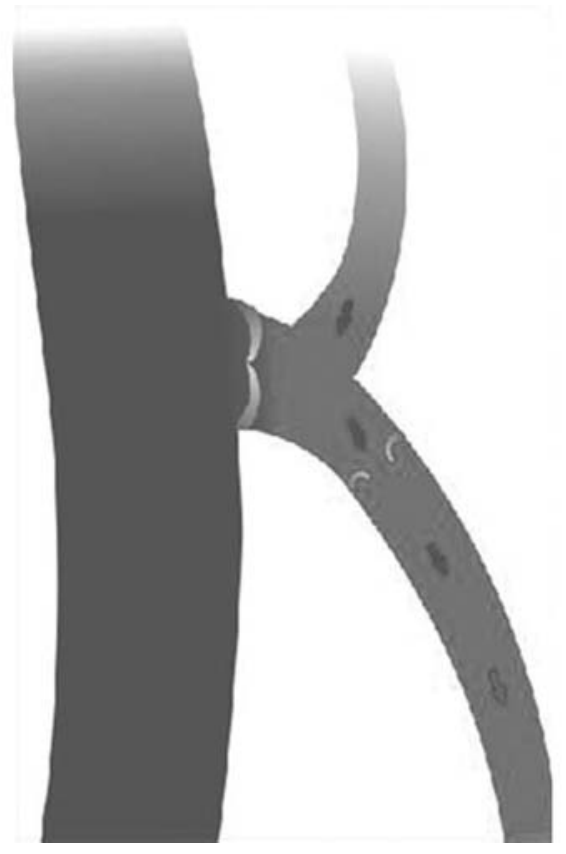

Figura 1 - Veia safena magna incompetente à custa de tributárias da crossa. A valva terminal é competente e a pré-terminal incompetente. As setas indicam o sentido do fluxo

A rede venosa linfonodal se apresenta sob a forma de veias tortuosas, normalmente entre 1 e $3 \mathrm{~mm}$ de diâmetro, subaponeuróticas, situadas entre a safena acessória anterior e a safena magna. Essas veias se conectam à safena magna ao longo dos seus $10 \mathrm{a} 15 \mathrm{~cm}$ proximais, e suas conexões craniais são difíceis de precisar pelo ultra-som, por seu pequeno calibre e tortuosidade, tendo frequentemente um trajeto translinfonodal, esse, sim,

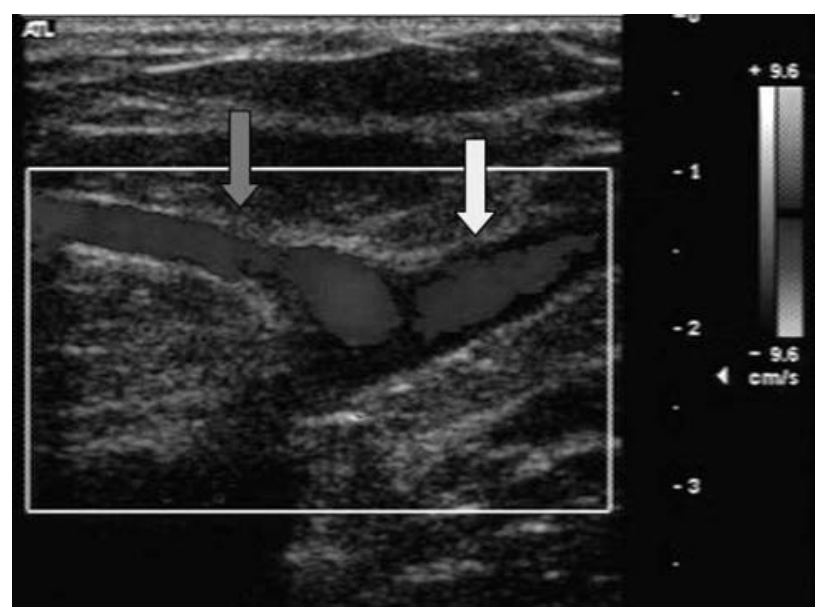

Figura 2 - Veia safena magna (seta branca) incompetente. A fonte do refluxo é a veia ilíaca circunflexa superficial (seta cinza) bem caracterizado ao estudo ultra-sonográfico e dopplerfluxométrico (Tabela 1, Figuras 3 e 4).

As veias linfonodais fazem parte de uma rede venosa no triângulo de Scarpa, que liga em vários pontos o sistema venoso superficial às veias profundas. Cranialmente, podem prolongar-se para as veias inguinoabdominais e pudendas externas e podem, ainda, perfurar a fáscia cribiforme conectando-se à veia femoral, fazendo o papel de pequenas perfurantes femorais diretas ${ }^{4}$. Tais veias já foram objeto de estudo anatômico em cadáveres, tendo sido caracterizadas em 19\% dos $\operatorname{casos}^{5}$.

\section{Veias linfonodais: aspectos hemodinâmicos}

Em uma série com 100 pacientes operados por incompetência da safena magna, Lemasle et al. encontraram veias linfonodais como causa principal do refluxo em $6 \%$ dos $\operatorname{casos}^{4}$. Essa frequência é provavelmente subestimada em estudos de causas de incompetência da safena magna por ser necessária uma pesquisa dirigida para sua adequada caracterização (Figura 5 e 6).

Mais bem definido é o seu papel na recorrência póssafenectomia. Alguns autores já tentaram associar a caracterização das veias linfonodais ectasiadas com neovascularização, inclusive sendo observadas, ao estudo histológico pós-operatório, veias linfonodais dispásicas que adentram nos linfonodos e penetram no tecido linfático $^{6-9}$. Porém, à luz do conhecimento anatômico atual, fica claro que se trata de hipertrofia de vasos préexistentes sob efeito de fatores angiogênicos (Figuras 7 , 8 e 9).

$\mathrm{Na}$ rotina diária dos exames de Doppler venoso de membros inferiores, temos notado de maneira cada vez mais freqüente a associação das veias linfonodais com varizes primárias e recorrentes.

\section{Conseqüências terapêuticas}

A recorrência de varizes é comum após a cirurgia envolvendo a veia safena magna. Taxas acima de $40 \%$ em 5 anos têm sido descritas, e aproximadamente $20 \%$ das cirurgias de varizes são realizadas visando a correção de veias recorrentes ${ }^{8,10}$. De acordo com a opinião prevalente, menor taxa de recorrência pode ser esperada com a fleboextração da veia safena magna, ligação 
Tabela 1 - Critérios ultra-sonográficos das veias linfonodais inguinais

Rede venosa tortuosa na topografia da lâmina linfonodal

Situada mais freqüentemente entre as veias safena acessória anterior e magna

Conecta-se ao tronco da veia safena magna (ou acessória anterior) ao longo dos seus 10 a $15 \mathrm{~cm}$ proximais

Trajeto translinfonodal

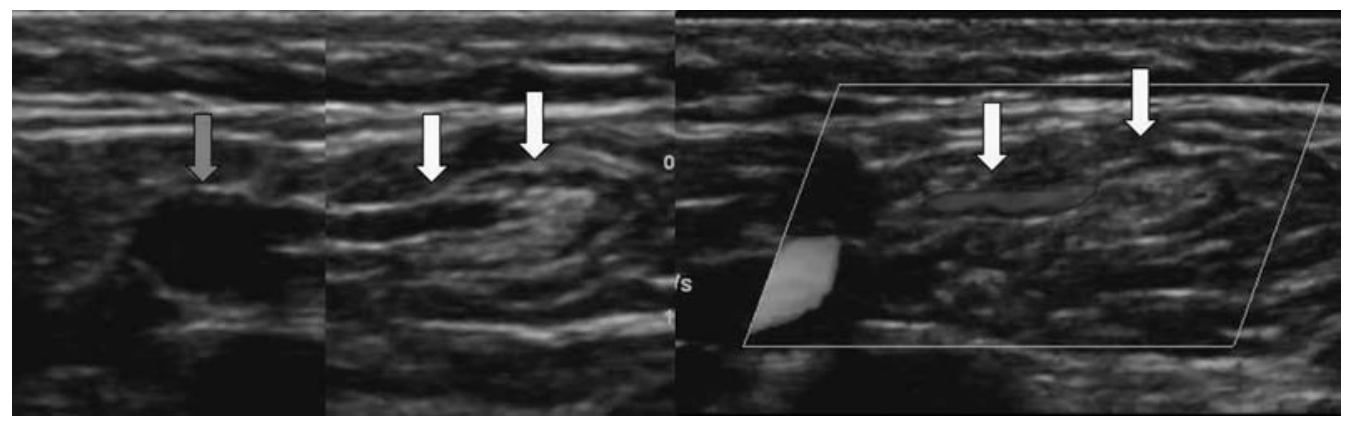

Figura 3 - Veia linfonodal (setas brancas) de aspecto habitual, drenando na crossa da veia safena magna (seta cinza)

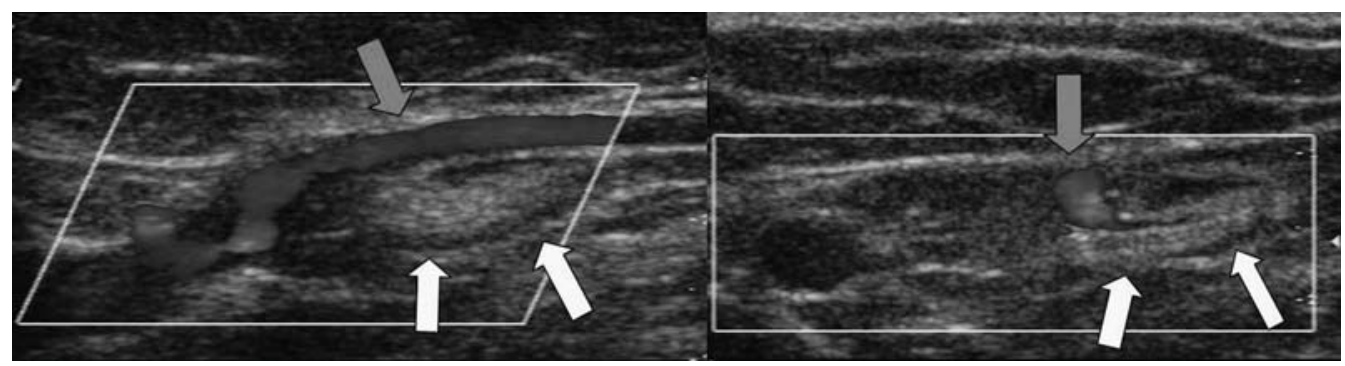

Figura 4 - Veia linfonodal de aspecto habitual (setas brancas) nos planos longitudinal e transversal, drenando na veia safena magna acessória anterior incompetente (setas cinza)

da junção safeno-femoral junto à veia femoral comum e interrupção de veias tributárias proximais ${ }^{7}$.

Dentre as principais fontes de refluxo comumente associadas à recorrência, destacam-se a persistência de coto residual incompetente, a neovascularização, veias perfurantes incompetentes não tratadas e tributárias residuais da crossa decorrente de tratamento cirúrgico incorreto. Tais achados justificam a ressecção estendida das tributárias da junção safeno-femoral, além de suas próprias tributárias primárias, visando uma desconexão mais efetiva, e evitando, assim, que vasos neoformados conectem os sistemas profundo e superficial ${ }^{6,11-14}$.
A abordagem cirúrgica das veias linfonodais é tecnicamente complicada e mesmo desaconselhável, já que são de pequeno calibre, friáveis e hemorrágicas, além de provavelmente deletério para o sistema linfático regional. Quando as veias linfonodais são a principal fonte de refluxo troncular da safena magna, estando a valva terminal competente, existe uma tendência a não se realizar a crossectomia, já que é inútil do ponto de vista hemodinâmico desconectar uma crossa competente. Além disso, sob o aspecto evolutivo, a agressão cirúrgica poderia desencadear um processo angiogênico sobre veias imaturas pré-existentes. O tratamento sugerido, 


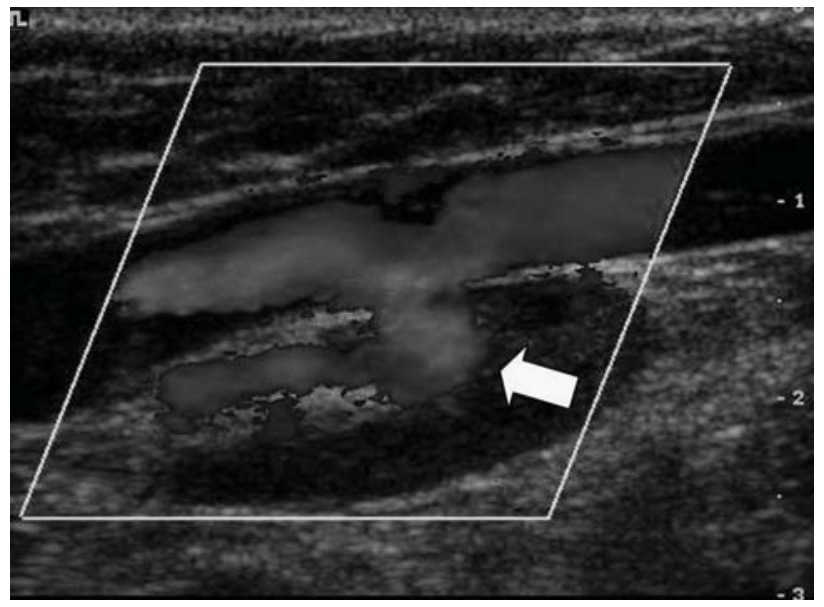

Figura 5 - Veia linfonodal (seta) como fonte de refluxo da veia safena magna

então, repousaria na destruição do tecido varicoso subjacente por flebectomia ou escleroterapia até o ponto da junção das veias linfonodais com a veia safena magna.

\section{Conclusão}

As veias linfonodais têm aspectos anatômicos e fisiológicos bem definidos. Porém, o seu verdadeiro papel como causa de varizes e sua abordagem mais adequada ainda estão por ser estabelecidos, já que carecem de maior número de estudos. Acreditamos que este artigo possa chamar a atenção dos profissionais envolvidos com o tema, colaborando com a discussão e elucidação da sua real importância.

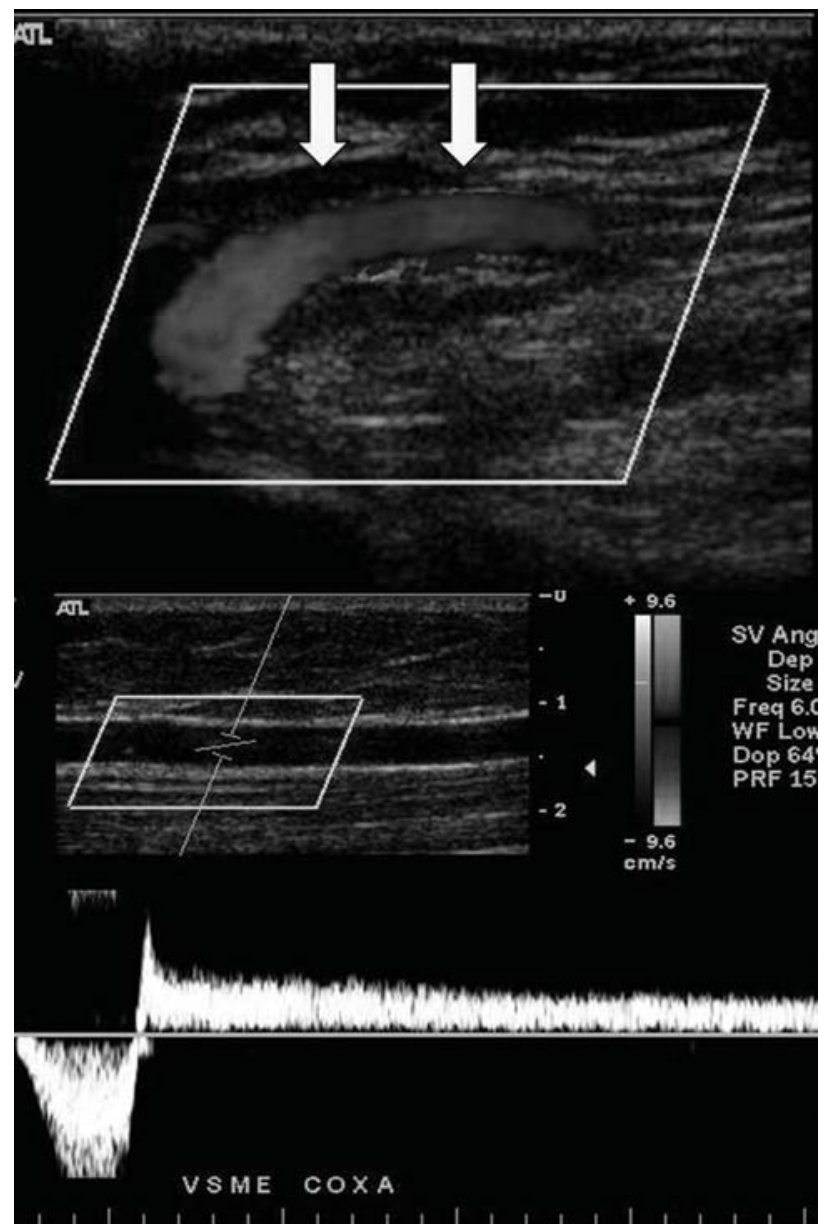

Figura 6 - Veia linfonodal ectasiada (setas) determina refluxo na veia safena magna

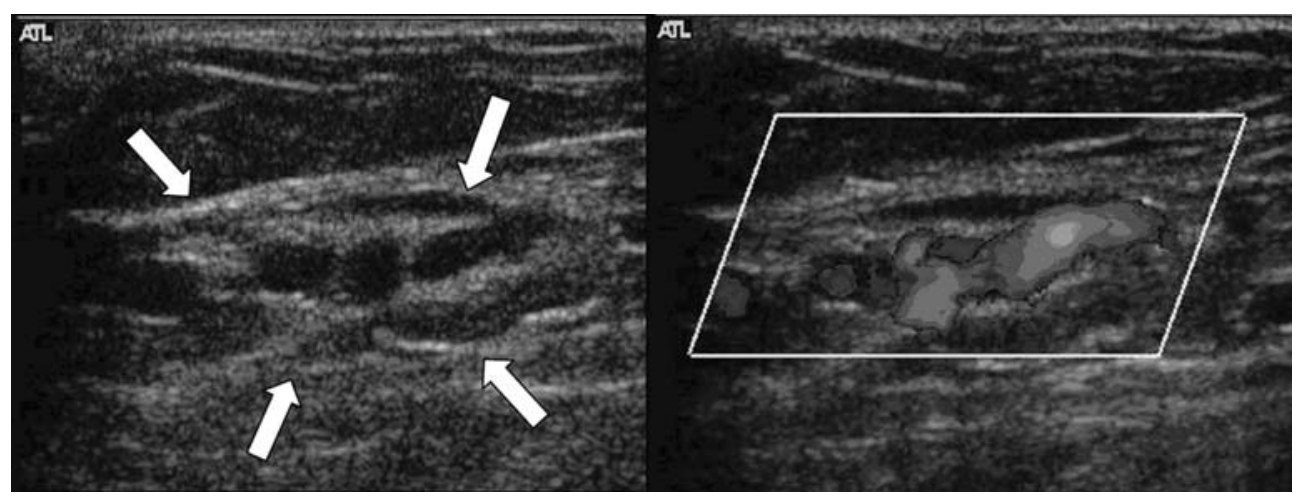

Figura 7 - Recorrência de varizes: veia linfonodal (setas) ingurgitando hilo e determinando varizes 


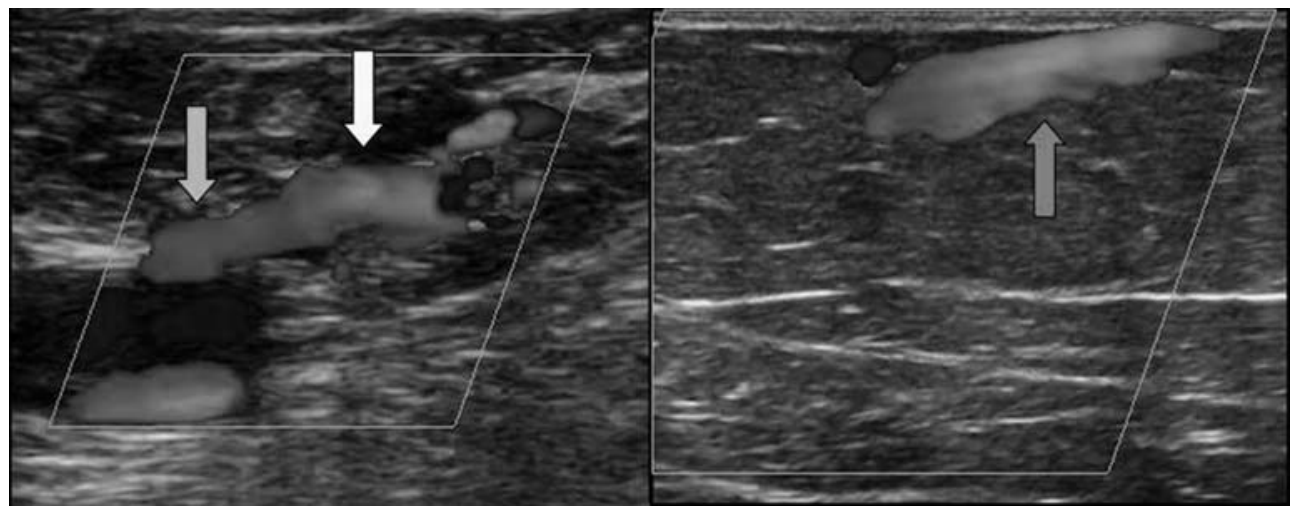

Figura 8 - A) Coto residual (seta cinza) drenando em veia linfonodal (seta branca) e B) determinando varizes recorrentes (seta cinza)

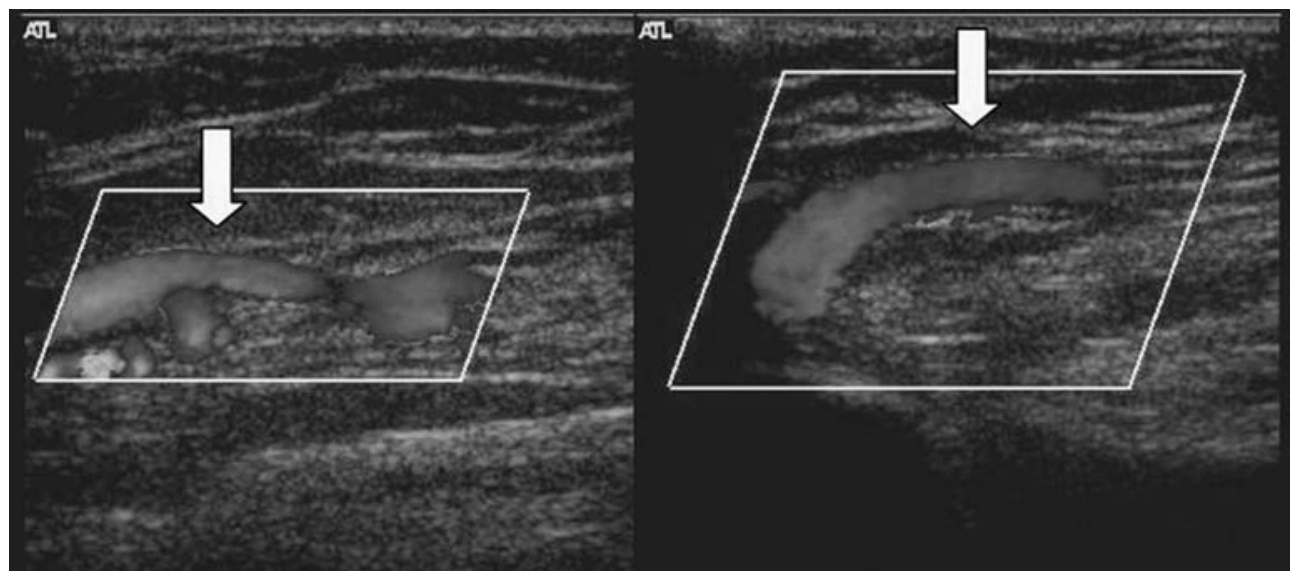

Figura 9 - Pós-operatório com varizes recorrentes à custa de veias linfonodais (setas)

\section{Referências}

1. Caggiati A, Bergan J, Gloviczki P, et al. Nomenclature of the veins of the lower limbs: an international interdisciplinary consensus statement. J Vasc Surg. 2002;36:416-22.

2. Caggiati A, Bergan J, Gloviczki P, et al. Nomenclature of the veins of the lower limbs: Extensions, refinements, and clinical application. J Vasc Surg. 2005;41:719-24.

3. Cavezzi A, Labropoulos N, Partsch H, et al. Duplex ultrasound investigation of the veins in chronic venous disease of the lower limbs-UIP consensus document. Part II. Anatomy. VASA. 2007;36:62-71.

4. Lemasle P, Uhl F, Lefrebve-Vilardebo M, Baud J, Gillot C. Veines lynpho-ganglionnaires inguinales. Aspects anatomiques et écographiques. Consequences sur la definition de la neogenese. Consequences therapeutiques. Phlebologie. 1999;52:263-269.

5. Guarrido M. A crossa da veia safena magna, estudo anatômico e correlação médico-cirúrgica. Tese de livre docência apresentada na Faculdade de Medicina Federal Fluminense Rio de janeiro, 1975.
6. Kohler A, Dirsch O, Brunner U. [Veno-lymphatic angiodysplasia as the cause of recurrent inguinal varicose veins]. VASA. 1997;26:52-4.

7. Franco G. La néovasculogenèse existe-t-elle? Récidives variqueuses post-chirurgicales. Point de vue d'um explorateur. Actualités Vasculaires Internat. 1992;1:41-3.

8. Franco G, Nguyen, K. Apport de l'echo-Doppler dans les récidives variqueuses post-opératoires au niveau de la région inguinale. Phlébologie. 1995;48:241-50.

9. Franco G. Exploration ultrasonographiche des récidives variqueuses post-chirurgicales. Phlébologie. 1998;51:403-13.

10. França GJ, Timi JRR, Vidal EA, Oliveira A, Secchi F, Miyamotto M. O eco-Doppler colorido na avaliação das varizes recidivadas. J Vasc Bras. 2005;4:161-6.

11. Chandler JG, Pichot O, Sessa C, Schuller-Petrovic S, Osse FJ, Bergan JJ. Defining the role of extended saphenofemoral junction ligation: a prospective comparative study. J Vasc Surg. 2000;32:941-53.

12. Fischer R, Linde N, Duff C, Jeanneret C, Chandler JG, Seeber P. Late recurrent saphenofemoral junction reflux after ligation and stripping of the greater sphenous vein. J Vasc Surg. 2001;34:236-40. 
13. Dwerryhouse S, Davies B, Harradine K, Earnshaw JJ. Stripping the long saphenous vein reduces the rate of reoperation for recurrent varicose veins: Five-year results of a randomized trial. J Vasc Surg. 1999;29:589-92.

14. Van Rij AM, Jiang P, Solomon C, Christie RA, Hill GB. Recurrence after varicose vein surgery: a prospective long-term clinical study with duplex ultrasound scanning and air plethysmography. J Vasc Surg. 2003;38:935-43.
Correspôndencia:

André Paciello Romualdo

Rua Martiniano de Carvalho, 836/32, bloco 1

01321-000 São Paulo, SP

Tel.: (11) 3283.0980

E-mail: andre.romualdo@fleury.com.br

\section{Aviso aos ex-residentes}

Se você foi residente até 2007 e ainda não está inscrito

em sua Regional, procure regularizar sua situação

para passar a receber o J Vasc Bras imediatamente, sem qualquer ônus.

Ajude a manter o cadastro da SBACV atualizado. 